\title{
Perfil de gestantes com distúrbios hipertensivos gestacionais e desenvolvimento de instrumento para qualificação do atendimento hospitalar
}

\author{
Profile of pregnant women with gestational hypertensive disturbances and development of \\ instrument to qualify hospital service \\ Perfil de gestantes con trastornos hipertensivos gestacionales y desarrollo de un instrumento para \\ calificar la atención hospitalaria
}

\begin{abstract}
Resumo
Distúrbios Hipertensivos Gestacionais (DHG) são as principais causas de morte materna, sendo necessário desenvolver meios de padronização e qualificação do atendimento hospitalar dessas gestantes e puérperas. Objetivo: Descrever o perfil de gestantes com DHG e o desenvolvimento de instrumentos para qualificação do cuidado hospitalar. Métodos: o estudo foi desenvolvido em uma instituição privada do sul do Brasil. O perfil das gestantes foi avaliado no período de janeiro a dezembro de 2017. Foram avaliadas as seguintes variáveis: PA, IG, tempo de internação, gestação anterior, prematuridade, reinternação, internação em CTI. Para qualificar o atendimento hospitalar foram desenvolvidos um protocolo institucional e uma carteirinha com orientações de alta para a puérpera. O desenvolvimento dos instrumentos baseou-se em grupos focais com profissionais especialistas e seguiu as seguintes etapas: definição de equipe de trabalho, revisão da literatura, identificação das necessidades de padronização e qualificação no atendimento; protótipo; ajustes; aprovação e implementação. Resultados: 3.458 gestantes internadas em 2017, 684 (19,78\%) apresentaram pressão arterial elevada durante a internação e $120(3,47 \%)$ desenvolveram DHG. Entre as alterações hipertensivas $(\mathrm{n}=684)$, a média de idade foi 31,6 anos; idade gestacional no parto em média 37,7 semanas, maioria primigesta $(n=377 ; 55,2 \%)$. O protocolo contemplou os seguintes itens: Padronização da Aferição da pressão arterial (PA); Conduta se PA elevada; Diagnóstico; Classificação; Fatores predisponentes; Critério para internação; Manejo; Interrupção da gestação; Alta hospitalar e medicamento na lactação. A carteirinha apresenta orientações sobre cuidados no puerpério. Conclusão: Complicações da HAS gestacional estendem-se ao puerpério. Os produtos qualificam a assistência.
\end{abstract}

Palavras-chave: Protocolos; Hipertensão induzida pela gravidez; Pré-eclâmpsia e eclampsia; Síndrome HELLP; Período pós-parto.

\begin{abstract}
DHG are the main causes of maternal death, and it is necessary to standardize and qualify assistance. Objective: to describe the profile of pregnant women with DHG and to develop instruments for qualifying care. Methods: it was developed in a private institution in southern Brazil. Period from January to December 2017. Variables: BP, IG, length of stay, previous pregnancy, prematurity, readmission, hospitalization in ICU. Protocol and discharge card elaborated. Steps: Team, literary review, needs; prototype; settings; approval and implementation. Results: 3,458 pregnant women hospitalized in 2017, 684 (19.78\%) had high BP and 120 (3.47\%) developed DHG. The changes $(\mathrm{n}=$ 684), mean age 31.6 years; GA at average delivery 37.7 weeks, primiparous $(n=377 ; 55.2 \%)$. The protocol included: Standardization of BP Measurement; Conduct; Diagnosis; Classification; Predisposing factors; Criterion for hospitalization; Management; Interruption of pregnancy; Discharge and medication. The card presents guidelines for the puerperium. Conclusion: Complications of gestational SAH extend to the puerperium. Products qualify for service.
\end{abstract}

Keywords: Protocols; Pregnancy-induced hypertension; Pre-eclampsia and eclampsia; HELLP syndrome; Postpartum period. 


\section{Resumen}

Los Trastornos Hipertensivos Gestacionales (DHG) sonlasprincipales causas de muerte materna, y esnecesariodesarrollar formas de estandarizar y calificarlaatenciónhospitalaria para estas embarazadas y mujeres que han dado a luz recientemente. Objetivo: Describirel perfil de gestantes con DHG y eldesarrollo de instrumentos para calificarlaatenciónhospitalaria. Métodos: elestudio se realizóen una institución privada delsur de Brasil. Se evaluóel perfil de las gestantes de enero a diciembre de 2017. Se evaluaronlassiguientesvariables: PA, EG, tiempo de estancia hospitalaria, embarazoprevio, prematurez, reingreso, ingresoen UCI. Para calificarlaatenciónhospitalaria se desarrollóun protocolo institucional y una ficha con pautas de alta para la puérpera. El desarrollo de los instrumentos se basóen grupos focalesconprofesionales especialistas y siguiólossiguientespasos: definicióndel equipo de trabajo, revisión de la literatura, identificación de lasnecesidades de estandarización y calificaciónenlaatención; prototipo; configuración; aprobación e implementación. Resultados: 3.458 embarazadas hospitalizadas en 2017, 684 (19,78\%) presentaronhipertensión arterial durante lahospitalización y 120 (3,47\%) desarrollaron DHG. Entre lostrastornos hipertensivos $(\mathrm{n}=684)$, laedad media fue de 31,6 años; edad gestacional al parto enpromedio 37,7 semanas, lamayoría de ellasprimigrávidas $(\mathrm{n}=377 ; 55,2 \%)$. El protocolo incluía lossiguientes elementos: estandarización de lamedición de lapresión arterial (PA); Conducir si la PA está alta; Diagnóstico; Clasificación; Factores predisponentes; Criterio de hospitalización; Administración; Interrupcióndelembarazo; Alta hospitalaria y medicación durante lalactancia. La tarjeta brinda orientación sobre laatenciónposparto. Conclusión: lascomplicaciones de la HSA gestacional se extienden hasta elpuerperio. Los productoscalifican para elservicio.

Palabras clave: Protocolos; Hipertensióninducida por elembarazo; Preeclampsia y eclampsia; Síndrome de HELLP; Período posparto.

\section{Introdução}

Todos os anos quase 76 mil mães e 500 mil bebês no mundo morrem por causa da pré-eclampsia, uma das complicações mais comuns entre os DHG. Essa doença afeta de $8 \%$ a $10 \%$ das gestações no mundo e responde por $20 \%$ de todas as hospitalizações para o tratamento intensivo neonatal. A hipertensão é a terceira causa principal de morte materna nos EUA, precedida apenas pelas hemorragias e infecções (ACOG, 2013). No Brasil, as principais causas de morte materna são pressão alta durante a gravidez, hemorragia após o parto, infecções e aborto. A mortalidade por hipertensão e suas complicações estão entre as maiores causas de morte materna e são causas evitáveis caso haja acompanhamento adequado no pré-natal e puerpério (Brasil, 2019).

A hipertensão arterial na gestação pode evoluir para quadros ainda mais complexos como pré-eclâmpsia, eclâmpsia, descolamento prematuro de placenta, síndrome de HELLP (haemolysis, elevatedliverenzymeactivity e lowplatelets), tromboembolismo, edema pulmonar, hemorragia cerebral, encefalopatia, CID (coagulação intravascular disseminada), insuficiência renal e hepática. Todas essas complicações são conhecidas como distúrbios hipertensivos gestacionais (DHG), sendo incuráveis, exceto pela interrupção da gravidez (Malachiaset al., 2016).

Segundo a $7^{\text {a }}$ Diretriz Brasileira de Hipertensão Arterial, define-se Hipertensão Arterial (HA) na gestação como a presença de Pressão Arterial Sistólica (PAS) $\geq 140$ mmHg e/ou Pressão Arterial Diastólica (PAD) $\geq 90$ mmHg, considerando-se o $5^{\circ}$ ruído de Korotkoff, confirmada por outra medida realizada com intervalo de 4 horas (Whelton et al., 2018). A hipertensão gestacional é caracterizada pela ocorrência de HA após a 20ª semana sem a presença de proteinúria (Malachiaset al., 2016). A hipertensão arterial crônica é definida pela detecção de HA precedendo a gestação, ou antes, da $20^{\mathrm{a}}$ semana. É possível que ocorra sobreposição com a doença gestacional (Malachiaset al., 2016). Pré-eclâmpsiaé definida por (PAS) $\geq 140$ ou (PAD) $\geq$ $90 \mathrm{mmHg}$ após a $20^{\mathrm{a}}$ semana gestacional, associada a edema significativo e presença de proteinúria. O diagnóstico de eclâmpsia se apresenta através de todos os sintomas e sinais que definem a pré-eclâmpsia, associado a crises convulsivas.

A pré-eclâmpsia grave ou eclâmpsia pode ser complicada por uma síndrome descrita pelo acróstico HELLP. Síndrome de HELLP é um grupo de sinais e sintomas, incluindo hemólise, elevação das enzimas hepáticas e contagem baixa de plaquetas. A etiologia dessa condição não está bem esclarecida, e não parece haver uma causa desencadeante (Laurenti, 2008). 
O American CollegeofCardiology e American Heart Associationpublicaram em sua última diretriz (2017) novos valores de referência para a classificação da hipertensão arterial em adultos. Os americanos inovaram e surpreenderam muita gente ao definir como PA ideal, associada a menor risco cardiovascular, valores <120/80 mmHg. Na nova diretriz, o diagnóstico de HAS que era dePAS $\geq 140 \mathrm{mmHg}$ e/ou PAD $\geq 90$ passa a ser PAS $\geq 130 \mathrm{mmHg}$ e/ou PAD $\geq 80 \mathrm{mmHg}$. No entanto, em relação ao rastreamento e diagnóstico de hipertensão gestacional, a nova diretriz americana não traz mudanças em relação aos pontos de corte previamente estabelecidos (Wheltonet al., 2018).

Embora o cuidado pré-natal adequado com as observações pertinentes para o controle da pressão arterial na gestante tenha reduzido o número e a extensão dos desfechos ruins - morbidade e mortalidade fetal e materna - a pré-eclâmpsia é considerada a principal causa de prematuridade entre os recém-nascidos americanos (ACOG, 2013).

No Brasil, segundo dados do Ministério da Saúde (MS), ela também é a principal responsável pelos nascimentos prematuros. Nesse contexto, as desordens hipertensivas representam um dos mais importantes problemas clínicos da gestação e estão associadas a altas taxas de morbimortalidade materna e perinatal no Brasil e no mundo (Brasil, 2019). No Rio Grande do Sul (RS), entre janeiro de 2008 a maio de 2019 ocorreram 42.647 internações no SUS relacionadas aos DHGs (CID10 O10O16). Sendo que 8.626 foram na cidade de Porto Alegre, o que representa 20,22\% das internações do estado. Em relação a mortalidade materna no RS entre 1996 e 2017 os DHGs foram responsáveis por 263 óbitos, sendo que Porto Alegre apresentou 31 casos (11,78\%) (Brasil, 2018).

Os gastos com internações decorrentes dos distúrbios hipertensivos na gestação são onerosos ao sistema. Dados do DATASUS sobre internações por hipertensão gestacional e suas complicações no Brasil, entre os anos 2008 e 2016, mostram mais de 711.332 internações e custos que ultrapassaram R\$ 500.000.000,00 no período (Malachias, 2016). Laurenti e colaboradores (2008), em pesquisa de base populacional, encontraram que as doenças hipertensivas foram responsáveis por um terço das causas diretas de morte materna no Brasil e que mais de 60\% dos óbitos ocorreram no puerpério.

Segundo o Global BurdenofDisease (GBD), que analisa dados dos níveis e tendências de saúde do mundo, apesar da queda na taxa de mortalidade materna nos últimos anos, a prevalência das doenças hipertensivas na gestação e suas complicações aumentou no Brasil cerca de 55,28\%, passando de 13,62 (IC 95\%: 9,45-18,75) para 21,15 (IC 95\%:14,52-28,81). No Rio Grande do Sul, esse aumento foi de 85,51\%, passando de 8,49 (IC 95\%: 5,8-12,08) para 15,75 (IC 95\%: 10,55-21,56) no mesmo período (IHME, 2018). Ou seja, os avanços da medicina e das políticas de saúde nos últimos 27 anos ocasionou a redução da mortalidade, mas não da incidência dos distúrbios.

Esses dados tornam-se ainda mais significativos quando olhamos para as taxas de natalidade e fertilidade no Brasil que, acompanhando uma tendência mundial, reduzindo nos últimos anos. A população continua aumentando, mas em menor velocidade. Dados do IBGE (2008) demonstram que na década de 1950, a taxa de natalidade no Brasil era de 44 nascimentos para cada mil habitantes, passando para 20,86 em 2000 e 14,16 em 2015, uma queda expressiva em poucas décadas. O mesmo ocorreu com a taxa de fecundidade que caiu 71\%, passando de 2,4 em 2000 para 1,72 filhos por mulher em 2015.

A identificação dos potenciais fatores de risco e a priorização de cuidados da equipe na assistência pré-natal, intraparto e pós-parto, são fundamentais para que se possa promover uma vigilância mais cuidadosa e identificar os sinais e sintomas da doença, minimizando intercorrências e um pior prognóstico entre essas pacientes.

\section{Métodos}

A execução do projeto ocorreu somente após autorização do mesmo pela instituição e aprovação no Comitê de Ética em Pesquisa do Hospital de Clínicas de Porto Alegre, sob o n ${ }^{\circ}$ CAAE 8938318700005327. Ele foi conduzido respeitando todos os preceitos éticos estabelecidos na Resolução nº 466/2012 do Conselho Nacional de Saúde (Brasil, 2013). 
A instituição onde o projeto foi desenvolvido denomina-se Sociedade Sulina Divina Providência (SSDP). É pessoa jurídica de direito privado, subespécie associação, de assistência à saúde, educacional, cultural, beneficente e de assistência social, com atividade preponderante na área da saúde, sem fins econômicos e lucrativos, constituída sob a inspiração dos ensinamentos e do carisma da Congregação das Irmãs da Divina Providência. É certificada como Entidade Beneficente de Assistência Social pelo Ministério da Saúde (Brasil, 2016).

A rede congrega cinco hospitais, além de inúmeros programas e projetos de assistência social em saúde. Com mais de 2.400 funcionários e 2.500 médicos credenciados, realiza em torno de 21.000 cirurgias ao ano, 160.000 atendimentos ambulatoriais e 99.000 exames. O Hospital Divina Providência, onde o estudo foi desenvolvido, atende apenas pacientes privados e que possuem planos de saúde.

$\mathrm{Na}$ etapa de definição do perfil da população de interesse foi realizado um estudo transversal, retrospectivo, de abordagem quantitativa (Pereira A.S. et al, 2018). Para elaboração do protocolo e da carteirinha foi realizada uma revisão de estudos mistos na literatura, combinando métodos que incluem componentes de revisão (habitualmente sistemáticos) com uma busca em estudos publicados nas áreas pertinentes à pesquisa, artigos e diretrizes, assim como o estabelecimento de vínculos com especialistas na área utilizando uma abordagem descritiva (Hulley, 2015)).

Para identificar o perfil das pacientes que apresentaram alteração de pressão arterial durante atendimento na instituição foram levantados dados no sistema de prontuários eletrônicos (Sistema TASY ${ }^{\circledR}$ ). A busca incluiu todas as mulheres internadas no Centro Obstétrico (CO) no ano de 2017. Foi realizada uma query no sistema para identificar as pacientes que apresentaram PA sistólica >140 mmHg ou diastólica >90 mmHg durante a internação. Observou-se dados sobre idade materna, idade gestacional, número de gestações anteriores, tempo de internação, presença de complicações graves relacionadas a elevação da pressão arterial durante a gestação (pré-eclâmpsia, eclâmpsia, síndrome de HELLP), parto prematuro, internação do recém-nascido em centro de terapia intensiva neonatal, internação da paciente em centro de terapia intensiva adulto e reinternação durante o puerpério.

Os dados foram tabulados em planilha de Excel. A análise do perfil das pacientes foi realizada no software SPSS ${ }^{\circledR}$ versão 18, através de estatística descritiva. Para comparar a proporção de alguns desfechos de interesse conforme a presença de DHG utilizou-se o teste exato de Fisher. As variáveis quantitativas foram descritas por média e desvio padrão ou mediana e amplitude interquartílica. As variáveis qualitativas foram descritas através de frequências absolutas e relativas.

\section{Etapas do Desenvolvimento dos Produtos}

A primeira etapa do desenvolvimento foi a identificação das necessidades de padronização e melhorias nos processos da instituição. Essa etapa ocorreu através da observação da pesquisadora, em sua rotina de trabalho, da necessidade de padronizar e qualificar a assistência às pacientes com DHG. A partir disso, surgiu a proposta de desenvolver um protocolo para gestantes com DHG e uma carteirinha de alta para puérperas. Essa proposta foi encaminhada para aprovação junto a liderança da área e direção do hospital. Após aprovadas ela seguiu para as demais etapas.

A segunda etapa foi a definição da equipe envolvida na elaboração e os procedimentos que seriam padronizados. Os profissionais envolvidos na equipe eram todos contratados, atuavam no centro obstétrico (CO) e alojamento conjunto da instituição e foram convidados pela diretoria que utilizou como critério a expertise dos mesmos. A equipe era composta pelo diretor assistencial, enfermeira líder do $\mathrm{CO}$, coordenador médico do $\mathrm{CO}$, um médico cardiologista que realiza acompanhamento cardiológico na unidade e a pesquisadora enfermeira. A definição dos procedimentos que seriam padronizados foi realizada em reunião presencial com todos os membros da equipe. 
A terceira etapa foi a definição do perfil das gestantes, cuja metodologia está sumarizada na sessão anterior, e a revisão da literatura para subsidiar o conteúdo dos produtos. Foi realizada uma revisão sistematizada na base de dados MEDLINE com os termos "hypertension" and "pregnancy" com filtro para "guidelines" dos últimos cinco anos. O critério de inclusão era ser diretriz ou posicionamento de sociedade nacional ou internacional que abordassem o tema hipertensão gestacional. Adicionalmente foi realizada busca não sistemática de artigos, livros sobre o assunto em estudo, e recomendações da Organização Mundial da Saúde.

A quarta etapa foi a elaboração do protocolo e da carteirinha e aprovação da equipe envolvida. A pesquisadora montou um protótipo do protocolo e da carteirinha e entregou para cada membro da equipe avaliar individualmente e fazer as suas contribuições. A quinta etapa foi a realização dos ajustes conforme as sugestões levantadas pela equipe. A pesquisadora considerou as contribuições e apresentou a versão ajustada em reunião presencial com todos os membros para aprovação final.

A sexta e última etapa foi a implementação do protocolo e da carteirinha e treinamento das equipes. Foram realizados dois treinamentos presenciais com técnicos de enfermagem, enfermeiros e médicos que atuam nos locais de aplicação dos produtos. Também foi disponibilizado o treinamento na versão EAD.

\section{Elaboração do Protocolo (Produto 1)}

O protocolo foi desenvolvido com o objetivo de qualificar o atendimento de pacientes que apresentam DHG na instituição. Para isso, se propôs padronizar as seguintes seções: identificação de gestantes hipertensas e classificação de acordo com os critérios diagnósticos atuais; verificação da gravidade do quadro clínico por meio de avaliações clínicas e laboratoriais necessárias; promoção do tratamento adequado para prevenir complicações clínicas maiores a curto e longo prazo tanto para a gestante quanto para o feto; redução do tempo de internação e custos hospitalares.

Os usuários do protocolo foram todos os profissionais dos setores envolvidos no cuidado às gestantes com DHG, contemplando médicos, enfermeiros e técnicos de enfermagem dos setores da emergência obstétrica, centro obstétrico, sala de recuperação obstétrica e alojamento conjunto.

O protocolo foi estruturado conforme o padrão da instituição, que contempla os seguintes itens: título, setor onde será aplicado, objetivo geral, introdução, objetivos específicos, lista de siglas, atividades essenciais, descrição do protocolo, fluxogramas, itens de controle e bibliografia.

\section{Carteirinha de Alta Hospitalar (Produto 2)}

A estruturação da carteirinha se deu por meio da seleção das principais recomendações de diretrizes sobre os cuidados necessários a puérperas que desenvolveram DHG. A mesma equipe envolvida na elaboração do protocolo revisou o conteúdo. A idealização desse produto se fez anteriormente ao propósito do protocolo. No entanto, a construção e a implementação dos mesmos ocorreram em conjunto, tornando-se complementares.

\section{Resultados}

No ano de 2017, foram internadas 3.458 gestantes no (CO) da instituição. Dessas, 684 (19,7\%) apresentaram pressão arterial superior a 140/90mmHg em algum momento durante a internação e compõem a amostra do estudo. Devido a heterogeneidade na forma de registro das informações em prontuário não foi possível definir qual a proporção de gestantes que possuíam diagnóstico prévio de HAS crônica ou gestacional. Foi possível avaliar o diagnóstico de complicações mais graves, como pré-eclampsia, eclampsia e síndrome HELLP. A presença dessas complicações ocorreu em 120 pacientes, o que corresponde a 3,4\% do total de atendimentos no CO em 2017. Esses resultados podem ser observados na Figura 1. 
Figura 1 - Presença de alteração de pressão arterial e DHG nas gestantes atendidas no HDP em 2017.

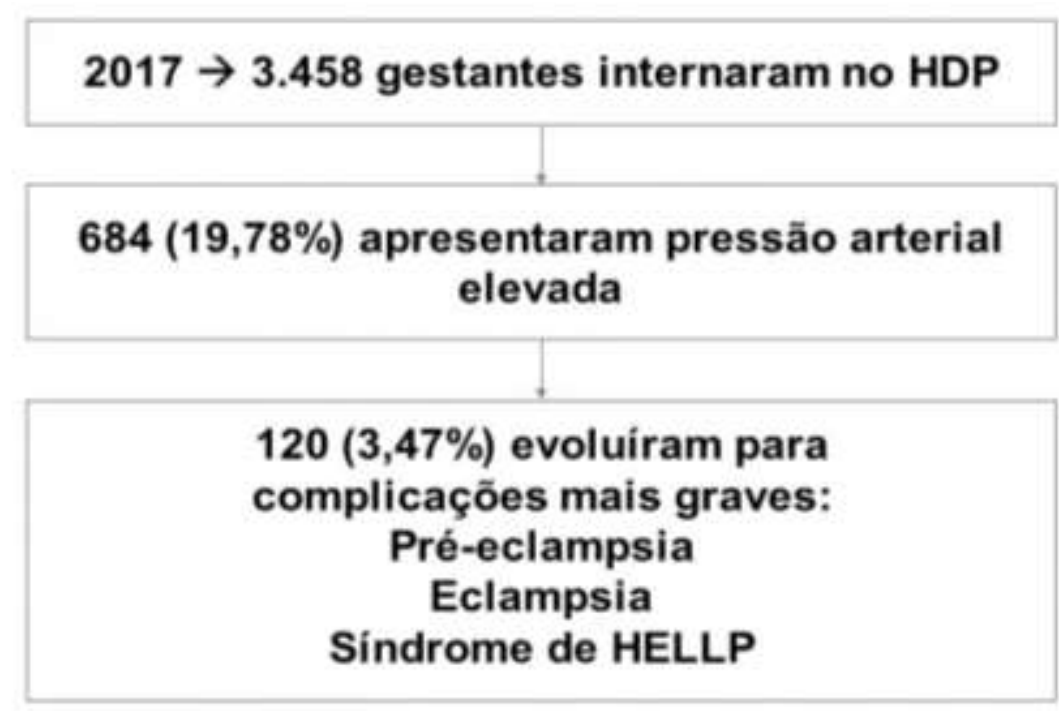

*Hospital Divina Providência (HDP). Fonte: Autores.

A média da idade geral da amostra de pacientes avaliadas foi $31,5( \pm 5,5)$ anos. A idade gestacional no momento do parto foi $37,7( \pm 2,1)$ semanas. O tempo mediano de internação foi de 54h:51min (49h:34min-66h:34min). Estavam na sua primeira gestação 377 (55,2\%) pacientes. A presença de DHG ocorreu em 120 pacientes, o que representa 17,5\% da amostra de pacientes que apresentaram elevação da PA em algum momento da internação. O DHG mais frequente foi a pré-eclâmpsia, com 111 casos (92,5\%), seguida de eclâmpsia com 2 casos (1,7\%) e HELLP com 7 casos (5,8\%).

Partos prematuros (<38 semanas) ocorreram em 184 pacientes e a internação do recém-nascido em unidade de terapia intensiva neonatal em 68 casos. Ocorreram 8 internações de puérperas em unidade de terapia intensiva e 48 reinternações durante o puerpério. A Tabela 1 descreve essas informações do perfil conforme a presença dos DHG com complicações. 
Tabela 1 - Perfil das pacientes que apresentaram PA $\geq 140 / 90 \mathrm{mmhg}$ em algum momento durante a internação ( $\mathrm{n}=684$ ), conforme presença de DHG.

\begin{tabular}{|c|c|c|c|c|}
\hline & \multicolumn{2}{|r|}{ DHG $\operatorname{sim}(n=120)$} & \multicolumn{2}{|r|}{ DHG não $(n=564)$} \\
\hline Variáveis & $\mathbf{n}$ & $\begin{array}{l}\text { Média (DP); n } \\
\text { (\%);mediana (AIQ) }\end{array}$ & $\mathbf{n}$ & $\begin{array}{l}\text { Média (DP); n (\%); } \\
\text { mediana (AIQ) }\end{array}$ \\
\hline Idade materna (anos) & 120 & $32,33(5,2)$ & 564 & $31,43(5,6)$ \\
\hline $\begin{array}{l}\text { Idade gestacional } \\
\text { momento parto (semanas) }\end{array}$ & 120 & $35,39(2,9)$ & 564 & $38,28(1,4)$ \\
\hline $\begin{array}{l}\text { Tempo de internação } \\
\text { (horas) }\end{array}$ & 120 & $73: 12(52: 23-129: 23)$ & 563 & $53: 55(49: 16-61: 27)$ \\
\hline Gestação anterior (sim) & 120 & $55(45,8 \%)$ & 563 & $251(44,6 \%)$ \\
\hline Parto prematuro (sim) & 108 & $78(72,2 \%)$ & 560 & $76(13,6 \%)$ \\
\hline $\begin{array}{l}\text { Internação do RN em CTI } \\
\text { neonatal (sim) }\end{array}$ & 110 & $50(45,5 \%)$ & 560 & $18(3,2 \%)$ \\
\hline $\begin{array}{l}\text { Internação da paciente em } \\
\text { CTI (sim) }\end{array}$ & 112 & $8(7,1 \%)$ & 564 & $0(0 \%)$ \\
\hline
\end{tabular}

$\begin{array}{lllll}\begin{array}{l}\text { Reinternação durante o } \\ \text { puerpério }(\operatorname{sim})\end{array} & 118 & 21(17,8 \%) & 564 & 27(4,8 \%)\end{array}$

*DP: desvio padrão; AIQ: amplitude interquartil; DHG: distúrbio hipertensivo gestacional; RN: recém-nascido; CTI: centro de terapia intensiva. Fonte: Autores (2019).

Ao comparar alguns desfechos, observou-se pior prognóstico naquelas que apresentaram DHG com complicações (pré-eclâmpsia, eclâmpsia e HELLP) em relação às demais. O parto prematuro ocorreu em 72,2\% das gestantes que desenvolveram DHG graves, enquanto apenas 13,6\% da amostra de pacientes que não apresentaram os distúrbios teve esse desfecho. Essa diferença foi estatisticamente significativa. A prevalência de casos de recém-nascido internados na CTI também foi significativamente maior entre os bebês de mães que apresentaram DHG (45,5\% vs 3,2\%).

Em relação a reinternação durante o puerpério observou-se que as gestantes que desenvolveram DHG com complicações tiveram maior taxa de reinternação $(17,8 \%)$ comparada com as pacientes que não desenvolveram $(4,8 \%)$, essa diferença também foi estatisticamente significativa. Em relação ao tempo de internação, as pacientes que apresentaram complicações mais graves tiveram uma mediana de tempo de internação de 73h:12min (52h:23min-129h:23min) enquanto entre as que não apresentaram a mediana foi de 53h:55min (49h:16min-61h:27min).

\section{Revisão da Literatura}

A busca na literatura foi realizada em julho de 2018 e resultou em 23 posicionamentos e diretrizes de sociedades da área, publicados nos últimos 5 anos. Após leitura de títulos e resumos 15 foram excluídas por não responderem questões dessa pesquisa ou por serem duplicadas. Oito diretrizes foram consideradas elegíveis e revisadas na íntegra. As recomendações foram utilizadas para subsidiar o desenvolvimento dos produtos da dissertação. 
O diagnóstico, classificação e orientações sobre o tratamento dos DHG eram muito semelhantes em todas as diretrizes. No entanto, as recomendações de cuidado pós-parto eram escassas. A maior parte delas abordou o tema de forma geral, sem fornecer orientações e recomendações de cuidado. Apenas 5 abordaram cuidados no puerpério com recomendações para verificação da pressão arterial de forma regular e manutenção de tratamento medicamentoso se necessário. Quatro forneceram recomendações para o pós-parto de médio a longo prazo (após o puerpério), sendo que as principais foram: prevenção de doença cardiovascular e renal, acompanhamento médico regular e mudança de estilo de vida (MEV). A tabela 6 apresenta o resumo das recomendações das diretrizes em relação aos cuidados no pós-parto imediato (puerpério) e em longo prazo.

\section{Discussão}

O perfil das pacientes e a prevalência de DHG encontrado neste estudo foram similares aos relatados na literatura. Em um hospital público de São Paulo, no período de janeiro a julho de 2002, que avaliou retrospectivamente 604 prontuários de gestantes internadas. Foram identificados 22 casos de DHG no período, o que representa 3,64\%. No estudo de São Paulo, 45,4\% eram adolescentes e 40,9\% primigestas, enquanto na nossa amostra a maior parte das gestantes eram adultas (média de $31,5 \pm 5,5$ anos) e $377(55,2 \%)$ das pacientes estavam em sua primeira gestação. Assim como observado nessa avaliação a complicação mais frequente foi a pré-eclâmpsia (Gonçalves, 2005).

Uma pesquisa realizada no Hospital Universitário Lauro Wanderley situado em João Pessoa analisou uma amostra de 62 gestantes com diagnóstico de DHG, no período de junho a setembro de 2018 e observou que 84\% das gestantes tiveram entre 1 a 3 gestações anteriores (Silva, 2018). Em nossa amostra, 44,7\% de mulheres não eram primigestas. Assim como nós, esse estudo também identificou a pré-eclâmpsia como o DHG mais frequente. Esses dados servem de alerta, visto que a préeclâmpsia é reconhecida como uma das principais causas para a morbidade e mortalidade materna e fetal em todo o mundo (Alvarez-Alvarez, 2107).

Outro estudo que avaliou 103 gestantes portadoras de hipertensão prévia e identificou que 25\% delas evoluíram para pré-eclâmpsia e/ou eclâmpsia. Esse achado reforça a importância de desenvolvermos instrumentos para rastrear, diagnosticar e tratar as gestantes com DHG de forma correta, a fim de evitar complicações maiores (Williams, 2018).

Um estudo transversal realizado em 2012, com o intuito de verificar a prevalência de complicações maternas e neonatais no parto de mães hipertensas, mostrou que essas síndromes levam a partos prematuros, ocasionando complicações imediatas nos neonatos, como baixo peso e necessidade de suporte ventilatório. Em uma amostra de 40 pacientes 35 (87,5\%) dos recém-nascidos, eram prematuros, 31 (77,5\%) eram de baixo peso e desses, 19 (47,5\%) eram pequenos para a idade gestacional (Gomes \&Gardenghi, 2013). Na nossa amostra, a prevalência de partos prematuros foi de $23 \%$ e a internação do recém-nascido em unidade de terapia intensiva neonatal ocorreu em 68 bebês (10\%). Essa menor prevalência em nosso estudo pode ser explicada pelo fato de não se tratar de amostra composta somente de pacientes previamente hipertensas, como na pesquisa citada.

Encontramos maior prevalência de reinternações durante o período do puerpério entre as gestantes que apresentaram DHG. Estudo realizado em um hospital do Pará identificou que dos óbitos maternos registrados no período avaliado, 81,8\% ocorreram no puerpério. Frente a isso, surge a preocupação referente ao planejamento da alta hospitalar, principalmente devido ao alto custo das internações, agravado pela falta de continuidade do cuidado domiciliar, acarretando reinternações e exposição ao risco de infecção da paciente durante a re-hospitalização (Guerreiro, 2014). Sendo assim, a garantia de continuidade dos cuidados iniciados no âmbito hospitalar depende das orientações fornecidas na alta, uma vez que contribui para a recuperação 
da paciente, minimiza a insegurança e proporciona melhor qualidade de vida familiar e social, além de prevenir complicações e/ou morbidades e evitar reinternações.

Além disso, a hipertensão gestacional constitui um risco materno, induzindo alterações metabólicas e vasculares a longo prazo, podendo estar associada ao aumento do risco cardiovascular (COP, 2015; Di Martino, 2016). Por isso, a gestante deve ter seu perfil de risco cardiovascular avaliado depois de 6 meses do parto e, sob orientação médica, iniciar medidas preventivas eficazes com a maior precocidade possível, evitando outras complicações (São Paulo, 2018). A partir dessa constatação, alguns estudos esclareceram que as mulheres grávidas com hipertensão são consideradas vulneráveis e necessitam de maior atenção de saúde, principalmente nos casos mais graves, exigindo um conhecimento maior dos profissionais responsáveis (Thuler, 2018).

Os cuidados assistenciais em relação às pacientes gestantes hipertensas requerem capacitação, habilidade e efetividade do profissional no manejo de situações que possam trazer complicadores durante o ciclo gravídico-puerperal. Logo, a elaboração e implementação de protocolos de atendimento são extremamente relevantes para a prática assistencial.

A enfermagem ocupa uma histórica e importante inserção, o que vem a exigir-lhe uma constante busca pela qualidade do cuidado prestado por seus profissionais. Essa qualidade é obtida não somente pelas vias de uma formação de qualidade, mas com a adoção de tecnologias e instrumentos que estabeleçam diretrizes que possam nortear e qualificar as suas práticas, como os protocolos (COFEN, 2108).

A elaboração de protocolos é a sistematização da assistência de enfermagem, que deve ser realizado em todo cuidado profissional de forma transversal, baseado em um suporte teórico organizado em cinco etapas inter-relacionadas, interdependentes e recorrentes, quais sejam: 1. Histórico de Enfermagem (coleta de dados); 2. Diagnóstico de Enfermagem; 3. Planejamento de Enfermagem; 4. Implementação; 5. Avaliação de Enfermagem (COFEN, 2108).

Apesar de não haver metodologia padronizada para o desenvolvimento de protocolos institucionais, e da escassez de dados referentes a implementação de protocolos de cuidado na literatura, o estudo desenvolvido no Hospital Divina Providência cumpriu com as exigências preconizadas pelo COFEN e conseguiu desenvolver e implementar um instrumento que está colaborando para as boas práticas no atendimento a gestante na instituição.

Mesmo bem estabelecido na literatura que o período do puerpério ainda traz risco de complicações derivadas da HAS gestacional e que essas gestantes possuem maior risco de desenvolver doenças cardiovasculares a médio e longo prazo, a maior parte das diretrizes das sociedades profissionais aborda o tema sem fornecer orientações e recomendações (Di Martino, 2016; COFEN, 2018). Apenas algumas abordam cuidados no puerpério de forma clara com recomendações para verificação da pressão arterial regular e manutenção de tratamento medicamentoso se necessário (ACOG, 2019; COP, 2015; Leung, 2016). Assim como poucas fornecem recomendações para cuidados no pós-parto de médio a longo prazo (Lowe, 2015). Baseado nisso, a criação da Carteirinha de Alta Hospitalar para cuidados e monitorização da pressão arterial domiciliar no puerpério foi desenvolvida para orientar pacientes e familiares sobre os riscos, na tentativa de minimizá-los a curto, médio e longo prazo. Ela surgiu para complementar o protocolo, preenchendo uma lacuna importante no seguimento da puérpera hipertensa, sendo um contribuinte às recomendações na alta hospitalar. Por fim, é preciso dar a devida atenção às condições que causam morbidade materna, especialmente aquelas condições que podem evoluir para morte se não for oferecido um cuidado adequado e oportuno. É necessário potencializar o uso dos recursos humanos e materiais existentes para um melhor enfrentamento desse problema (São Paulo, 2018). Tendo em vista a multiplicidade de fatores predisponentes ao desenvolvimento do DHG, a revisão da literatura e das diretrizes norteadoras de técnicas seguras foram imprescindíveis para o desenvolvimento dos produtos dessa dissertação. Esse trabalho solidificou os pilares de atendimento às gestantes sob risco na instituição Hospital Divino Providência através dos produtos construídos e implementados. 
Durante o desenvolvimento do trabalho houve algumas barreiras que limitaram os resultados do estudo do perfil das pacientes, por exemplo: não foi possível fazer um levantamento do nível de escolaridade das gestantes por registros insuficientes e dificuldades de captar a informação de forma automatizada no sistema; a maioria dos prontuários não apresentavam informações sobre presença de hipertensão prévia à gestação. A falta de registros padronizados também dificultou a busca por desfechos e absorveu um maior tempo da pesquisadora visto que foi necessário fazer uma busca mais detalhada dentro dos registros disponíveis.

Além disso, não foi possível avaliar mudanças nos desfechos após a implementação dos produtos da dissertação devido a saída da pesquisadora da instituição em dezembro de 2018, dois meses após a implementação dos produtos.

Os produtos apresentaram potencialidades importantes, inovando a rotina de atendimento dentro da instituição. $\mathrm{O}$ protocolo tornou-se uma ferramenta para a educação permanente dos colaboradores envolvidos no atendimento de gestantes com DHG. A carteirinha da puérpera passou a ser uma importante fonte de educação em saúde para as mulheres atendidas no HDP. Ambos os produtos têm potencial para serem implementados em outras instituições.

\section{Conclusão}

No estudo em questão foi verificado que a pressão arterial elevada foi fator de repercussão no desencadeamento de complicações na gestação, resultando em perigo para a gestante e para o feto. O protocolo implementado propôs padronização das práticas assistenciais, tornando-se um instrumento adequado e útil para a melhoria da qualidade no atendimento às pacientes e garantindo um manejo mais ágil no centro obstétrico. A carteirinha de alta hospitalar com suas recomendações é um instrumento educativo para prevenir complicações no período pós-parto. A implementação dos produtos provenientes desse trabalho propiciou à instituição qualificação do atendimento e dos profissionais envolvidos no cuidado à gestante e puérpera. Esta pesquisa nos proporciona a possibilidade de novos estudos a ser desenvolvidos a partir deste, com o objetivo de mensurar os resultados obtidos após a implementação dos produtos.

\section{Referências}

Alvarez-Alvarez, B., Martell-Claros, N., Abad-Cardiel, M., \& García-Donaire, J. A. (2017). Trastornos hipertensivos enelembarazo: repercusión a largo plazoenlasalud cardiovascular de lamujer. Hipertension y riesgo vascular, 34(2), 85-92. https://doi.org/10.1016/j.hipert.2016.06.002.

American College of Obstetricians and Gynecologists [ACOG] (2019).Clinical management guidelines for obstetrician - chronic hypertension in pregnancy, Obstetrics and gynecology, 133(1), 26-50.

American College of Obstetricians and Gynecologists [ACOG], \& Task Force on Hypertension in Pregnancy (2013). Hypertension in pregnancy.Report of the American College of Obstetricians and Gynecologists Task Force on Hypertension in Pregnancy.Obstetrics and gynecology, 122(5), 1122-1131. https://doi.org/10.1097/01.AOG.0000437382.03963.88.

Brasil.Ministério da Saúde (2013). Resolução $\mathrm{n}^{\circ}$ 466, de 12 de dezembro de 2012. Diretrizes e normas regulamentadoras de pesquisas envolvendo seres humanos. Recuperado em http://bvsms.saude.gov.br/bvs/saudelegis/cns/2013/res0466_12_12_2012.html.

Brasil. Ministério da Saúde (2016). Portaria $\mathrm{n}^{\circ}$ 834, de 26 de abril de 2016. Redefine os procedimentos relativos à certificação das entidades beneficentes de assistência social na área de saúde. Recuperado em http://bvsms.saude.gov.br/bvs/saudelegis/gm/2016/prt0834_26_04_2016.html.

Brasil. Ministério da Saúde (2018). DATASUS. http://www2.datasus.gov.br/DATASUS/index.php?area=0203\&id=6926.

Committee on Obstetric Practice [COP] (2015). Committee Opinion No. 623: Emergent therapy for acute-onset, severe hypertension during pregnancy and the postpartum period. Obstetricsandgynecology, 125(2), 521-525. https://doi.org/10.1097/01.AOG.0000460762.59152.d7.

Conselho Federal de Enfermagem [COFEN] (2018). Diretrizes para elaboração de protocolos de enfermagem na atenção primária à saúde pelos conselhos regionais. Brasília: COFEN.

Di Martino, D., Cetin, I., Frusca, T., Ferrazzi, E., Fuse', F., Gervasi, M. T., Plebani, M., \&Todros, T. (2016). Italian Advisory Board: sFlt-1/P1GF ratio and preeclampsia, state of the art and developments in diagnostic, therapeutic and clinical management. European journal of obstetrics, gynecology, and reproductive biology, 206, 70-73. https://doi.org/10.1016/j.ejogrb.2016.08.036.

Gomes, N. R., \&Gardenghi, G. (2013). Prevalência de complicações maternas e neonatais em gestações que apresentaram síndromes hipertensivas. Revista 
Research, Society and Development, v. 10, n. 2, e2701028913, 2021

(CC BY 4.0) | ISSN 2525-3409 | DOI: http://dx.doi.org/10.33448/rsd-v10i2.8913

Eletrônica Saúde e Ciência, 3 (1), 2230-4111.

Gonçalves, R., Fernandes, R. A., \& Sobral, D. H. (2005). Prevalência da doença hipertensiva específica, da gestação em hospital público de São Paulo. Revista brasileira de enfermagem, 58(1), 61-64. https://doi.org/10.1590/s0034-71672005000100011.

Guerreiro, D. D., Borges, W. D., de Moura Nunes, H. H., Silva, S. C. S., \& de Paula Maciel, J. (2014). Mortalidade materna relacionada à doença hipertensiva específica da gestação (DHGE) em uma maternidade no Pará. Revista de Enfermagem da UFSM, 4(4), 825-834.

Hulley, S. B., Cummings, S. R., Browner, W. S., Grady, D. G., \& Newman, T. B. (2015). Delineando a pesquisa clínica-4. Artmed.

Institute for Health Metrics and Evaluation [IHME] (2018). Global BurdenofDisease. http://www.healthdata.org/gbd.

Instituto Brasileiro de Geografia e Estatística [IBGE] (2008). Revisão 2008 - projeção da população - taxas brutas de natalidade. https://seriesestatisticas.ibge.gov.br/series.aspx?vcodigo=POP318.

Laurenti, R., Jorge, M. H. P., \&Gotlieb, S. L. D. (2008). Mortes maternas e mortes por causas maternas. Epidemiologia e Serviços de Saúde, 17(4), 283-292.

Leung, A. A., Nerenberg, K., Daskalopoulou, S. S., McBrien, K., Zarnke, K. B., Dasgupta, K., Cloutier, L., Gelfer, M., Lamarre-Cliche, M., Milot, A., Bolli, P., Tremblay, G., McLean, D., Tobe, S. W., Ruzicka, M., Burns, K. D., Vallée, M., Prasad, G. V., Lebel, M., Feldman, R. D., ... CHEP Guidelines Task Force (2016). Hypertension Canada's 2016 Canadian hypertension education program guidelines for blood pressure measurement, diagnosis, assessment of risk, prevention, and treatment of hypertension.The Canadian journal of cardiology, 32(5), 569-588. https://doi.org/10.1016/j.cjca.2016.02.066.

Lowe, S. A., Bowyer, L., Lust, K., McMahon, L. P., Morton, M., North, R. A., Paech, M., \& Said, J. M. (2015). SOMANZ guidelines for the management of hypertensive disorders of pregnancy 2014.The Australian \& New Zealand journal of obstetrics \&gynaecology, 55(5), e1-e29. https://doi.org/10.1111/ajo.12399.

Malachias, M. V. B., Figueiredo, C. E. P., Sass, N., Antonello, I. C., Torloni, M. R., \&Bortolotto, M. R. F. L. (2016). $7^{a}$ Diretriz Brasileira de Hipertensão Arterial: Capítulo 9-Hipertensão arterial na gestação. Arquivos Brasileiros de Cardiologia, 107(3), 49-52.

Mounier-Vehier, C., Amar, J., Boivin, J. M., Denolle, T., Fauvel, J. P., Plu-Bureau, G., Tsatsaris, V., \&Blacher, J. (2016).Hypertensionartérielleetgrossesse. Consensus d'experts de laSociétéfrançaise d'hypertensionartérielle, filiale de laSociétéfrançaise de cardiologie. Presse medicale, 45(7-8 Pt 1), 682-699. https://doi.org/10.1016/j.lpm.2016.05.012.

Rodrigues C. I. (2016). Hipertensão crônica na gestação: muito a aprender. Jornal brasileiro de nefrologia, 38(2), 143-144. https://doi.org/10.5935/01012800.20160021 .

Pereira, A. S., Shitsuka, D. M., Pereira, F. J., \& Shitsuka, R. Metodologia do trabalho científico. UFSM.

São Paulo. Secretaria da Saúde (2018). Atenção à gestante e à puérpera no SUS - SP: manual de orientação ao gestor para implantação da linha de cuidado da gestante e da puérpera. São Paulo: SES/SP.

Silva, C. S. D. (2018). Perfil de risco gestacional e desfechos maternos em mulheres com síndromes hipertensivas. Monografia. Universidade Federal da Paraíba, João Pessoa, PB, Brasil.

Silva, E. R. A. D. C. (2018). Agenda 2030: ODS-Metas nacionais dos objetivos de desenvolvimento sustentável. Brasília: IPEA.

Thuler, A. C. D. M. C., Wall, M. L., Benedet, D. C. F., Souza, S. R. R. K., \& Souza, M. A. R. D. (2018). Medidas preventivas das síndromes hipertensivas da gravidez na atenção primária. Revista de enfermagem UFPE online, 12(4), 1060-1071.

Whelton, P. K., Carey, R. M., Aronow, W. S., Casey, D. E., Jr, Collins, K. J., Dennison Himmelfarb, C., DePalma, S. M., Gidding, S., Jamerson, K. A., Jones, D. W., MacLaughlin, E. J., Muntner, P., Ovbiagele, B., Smith, S. C., Jr, Spencer, C. C., Stafford, R. S., Taler, S. J., Thomas, R. J., Williams, K. A., Sr, Williamson, J. D., Wright, J. T., Jr (2018). 2017 ACC/AHA/AAPA/ABC/ACPM/AGS/APhA/ASH/ASPC/NMA/PCNA Guideline for the Prevention, Detection, Evaluation, and Management of High Blood Pressure in Adults: a report of the American College of Cardiology/American Heart Association Task Force on Clinical Practice Guidelines. Journal of the American College of Cardiology, 71(19), e127-e248. https://doi.org/10.1016/j.jacc.2017.11.006.

Williams, B., Mancia, G., Spiering, W., AgabitiRosei, E., Azizi, M., Burnier, M., Clement, D. L., Coca, A., de Simone, G., Dominiczak, A., Kahan, T., Mahfoud, F., Redon, J., Ruilope, L., Zanchetti, A., Kerins, M., Kjeldsen, S. E., Kreutz, R., Laurent, S., \& Lip, G. ESC Scientific Document Group (2018). 2018 ESC/ESH Guidelines for the management of arterial hypertension. European heart journal, 39(33), 3021-3104. https://doi.org/10.1093/eurheartj/ehy339. 